\title{
Special issue on data analysis methodologies for intelligent systems: guest editor's introduction
}

\author{
Tapio Elomaa · Petr Berka $\cdot$ Jan Rauch
}

Received: 18 August 2011 / Accepted: 22 August 2011/

Published online: 15 September 2011

(C) Springer Science+Business Media, LLC 2011

Modern data analytics enables one to meaningfully interpret the masses of raw data that is increasingly available to us through the advanced data gathering tools. Furthermore, increased computational power and ever more sophisticated algorithms let us reveal information that previously was inaccessible to us. The articles in this special issue all approach data analysis methodologies for intelligent systems from their individual perspectives, but together paint a picture of the power provided by empirical and analytical tools for building the intelligent systems of tomorrow. The application domains from which data is gathered are numerous, as are the formats in which the data is presented. The articles in this special issue cover, e.g., analysis of multi-instrument music data, relational database queries, graph data, and time series data.

Alicja A. Wieczorkowska and Agnieszka Kubik-Komar deal with distinguishing between musical instruments depending on the chosen sound features in their article Application of Analysis of Variance and Post Hoc Comparison to Studying the Discriminative Power of Sound Parameters in Distinguishing between Musical Instruments. The paper On Three Classes of Division Queries Involving Ordinal

T. Elomaa $(\varangle)$

Department of Software Systems, Tampere University of Technology,

P.O. Box 553, Korkeakoulunkatu 1, 33101 Tampere, Finland

e-mail: elomaa@cs.tut.fi

P. Berka · J. Rauch

University of Economics, Prague W. Churchill Sq. 4, 13067 Prague, Czech Republic

P. Berka

e-mail: berka@vse.cz

J. Rauch

e-mail: rauch@vse.cz 
Preferences by Patrick Bosc, Olivier Pivert, and Olivier Soufflet takes preferences into account for a family of division queries in relational databases.

Guillaume Poezevara, Bertrand Cuissart, and Bruno Crémilleux in their article Extracting and Summarizing the Frequent Emerging Graph Patterns from a Dataset of Graphs are concerned with mining emerging patterns from graph data, in particular real-world chemicals. Visualization of Trends Using RadViz by Lenka Nováková and Olga Štěpánková deals with typical qualitative development patterns in time series data. Finally, SEWEBAR-CMS: Semantic Analytical Report Authoring for Data Mining Results by Tomáš Kliegr, Vojtěch Svátek, Martin Ralbovský and Milan Šmůnek puts forward a communication platform between the data analyst, domain expert, and the content management system report user.

We are most pleased to present these five articles whose initial manuscripts were presented at the 18th International Symposium on Methodologies for Intelligent Systems (ISMIS 2009) held in Prague, Czech Republic, September 14-17, 2009. The authors of selected papers were invited to submit a substantially enhanced version of their paper to this special issue, each paper was reviewed and further revised by the authors. ISMIS is an established and prestigious conference for exchanging the latest research results in building intelligent systems. Held twice every three years, the conference provides a medium for exchanging scientific research and technological achievements accomplished by the international community. The scope of ISMIS is intended to represent a wide range of topics on applying Artificial Intelligence techniques.

We wish to express our thanks to all authors for the excellent quality of their papers. We also thank to the reviewers of these papers for their comments and all other people that have contributed to this special issue. 